\title{
Continuous star formation in IZw18
}

\author{
S. Recchi ${ }^{1,2}$, F. Matteucci ${ }^{3}$, A. D’Ercole ${ }^{4}$, and M. Tosi ${ }^{4}$ \\ 1 Institut für theoretische Physik und Astrophysik, Kiel University, Olshausenstrasse 40, 24118 Kiel, Germany \\ e-mail: recchi@mpa-garching.mpg.de \\ 2 Max-Planck Institut für Astrophysik, Karl-Schwarzschild-Strasse 1, 85741 Garching bei München, Germany \\ 3 Dipartimento di Astronomia, Università di Trieste, Via GB Tiepolo, 11, 34131 Trieste, Italy \\ e-mail: matteucci@ts.astro.it \\ ${ }^{4}$ INAF - Osservatorio Astronomico di Bologna, Via Ranzani 1, 40127 Bologna, Italy \\ e-mail: [annibale; tosi]@abo.astro.it
}

Received 27 March 2004 / Accepted 25 May 2004

\begin{abstract}
We study the dynamical and chemical evolution of a galaxy similar to IZw18 under the assumption of a continuous star formation during bursts. We adopt a 2-D hydrocode coupled with detailed chemical yields originating from SNeII, SNeIa and from single intermediate-mass stars. Different nucleosynthetic yields and different IMF slopes are tested. In most of the explored cases, a galactic wind develops, mostly carrying out of the galaxy the metal-enriched gas produced by the burst itself. The chemical species with the largest escape probabilities are $\mathrm{Fe}$ and $\mathrm{N}$. Consequently, we predict that the $[\alpha / \mathrm{Fe}]$ and $[\alpha / \mathrm{N}]$ ratios outside the galaxy are lower than inside. In order to reproduce the chemical composition of IZw18, the best choice seems to be the adoption of the yields of Meynet \& Maeder (2002) which take into account stellar rotation, although these authors do not follow the whole evolution of all the stars. Models with a flat IMF $(x=0.5)$ seem to be able to better reproduce the chemical properties of IZw18, but they inject in the gas a much larger amount of energy and the resulting galactic wind is very strong, at variance with observations. We also predict the evolution of the abundances in the $\mathrm{H}$ i medium and compare them with recent FUSE observations.
\end{abstract}

Key words. hydrodynamics - ISM: abundances - ISM: jets and outflows - galaxies: evolution - galaxies: individual: IZw18 galaxies: spiral

\section{Introduction}

Blue Compact Dwarf (BCD) galaxies, due to their very blue colors, compact appearance, high gas content and low metallicities, are generally thought to be unevolved systems. With an oxygen abundance $(\mathrm{O} / \mathrm{H}) \sim 0.02(\mathrm{O} / \mathrm{H})_{\odot}$, IZw18 (Mrk 116) is the BCD galaxy with the lowest known metallicity as measured by nebular emission lines and the ideal candidate for a truly young local galaxy. This very low metal content was first observed by Searle \& Sargent (1972), then confirmed by many studies (e.g. Lequeux et al. 1979; French 1980; Kinman \& Davidson 1981; Kunth \& Sargent 1983; Davidson \& Kinman 1985; Davidson et al. 1989; Pagel et al. 1992; Skillman \& Kennicutt 1993; Thuan et al. 1995; Legrand et al. 1997; Vílchez \& Iglesias-Páramo 1998). Since its discovery (Zwicky 1966; Sargent \& Searle 1970), this galaxy has been extensively studied and nowadays we know with reasonable accuracy the chemical composition of the $\mathrm{H}_{\text {II }}$ regions (see e.g. Skillman \& Kennicutt 1993; Garnett et al. 1995; Izotov \& Thuan 1999, among others). IZw18 is thus a good benchmark to study the impact of starburst(s) on the ISM and compare the results of models with detailed metallicity determinations.
The determination of the metallicity in the $\mathrm{H}_{\mathrm{I}}$ regions is much more difficult, since the O I line at $1302 \AA$ is already strongly saturated at $N_{\mathrm{HI}} \gtrsim 2 \times 10^{20} \mathrm{~cm}^{-2}$ (the column density of IZw18 is larger than $10^{21} \mathrm{~cm}^{-2}$; van Zee et al. 1998). The first determination of $\mathrm{H}_{\mathrm{I}}$ metallicity content made by Kunth et al. (1994) (an abundance in the H I medium 30 times lower than the metallicity of the $\mathrm{H}$ II regions) was therefore uncertain and has been disputed by Pettini \& Lipman (1995). Recent FUSE (Far Ultraviolet Spectroscopic Explorer) data (Aloisi et al. 2003; Lecavelier des Etangs et al. 2004) allowed observations of $\mathrm{O}_{\mathrm{I}}$ lines at shorter wavelengths and with smaller oscillation strengths. The knowledge of the metallicity of the neutral medium has therefore been improved, but uncertainties are still present. Aloisi et al. (2003) and Lecavelier des Etangs et al. (2004), analyzing the same FUSE data, obtained different results. Aloisi et al. (2003) found abundances of various elements a factor of 3-10 lower than those found in the $\mathrm{H}$ II regions, with the exception of iron, whose abundance is the same. Lecavelier des Etangs et al. (2004) instead found an oxygen abundance consistent with the one found in the $\mathrm{H}$ II regions, whereas $\mathrm{N}$ and $\mathrm{Ar}$ are found to be much more underabundant.

The study of starburst and BCD galaxies by means of hydrodynamical simulations has been performed by several 
authors in the recent past. The overall picture is that galactic winds, produced by the combined effect of SNe explosions and stellar winds, are not able to eject a significant fraction of the ambient Interstellar Medium (ISM), since, due to the flattened distribution of the gas, the wind develops only in the polar direction (where the pressure gradient is steeper), whereas the gas in the disc is almost unperturbed. The final fate of the metals is still debated. Many authors (De Young \& Heckman 1994; D’Ercole \& Brighenti 1999; MacLow \& Ferrara 1999) believe that the metals are easily channelled along the funnel created by the galactic wind, so they are ejected outside the galaxy more easily than the pristine gas. Other authors (Silich \& Tenorio-Tagle 1998; Legrand et al. 2001) have suggested that the metal-rich gas is barely lost from the galaxies, since it is trapped by extended halos surrounding the galaxies, although the existence of such halos is still unproved. Rieschick $\&$ Hensler (2000) have suggested that $\sim 25 \%$ of the metals can mix rapidly, remaining trapped in the galaxy, whereas the other $75 \%$ of metals undergoes a cycle lasting $\sim 1 \mathrm{Gyr}$, in which an outflow is created. Cooling creates condensation of the metalrich gas into molecular droplets, able to fall back and settle onto the disc of the galaxy.

These studies do not focus on any specific object, trying to study general properties of starburst and BCD galaxies. There are a few exceptions: Strickland \& Stevens (1999) for NGC 5253 and Strickland \& Stevens (2000) for M82 in particular. More recently, Vorobyov et al. (2004) simulated the expansion of supershells in a model galaxy resembling Holmberg I. These authors tried to constrain in particular the gravitational potential and the number of SNeII needed to reproduce the observed Hi morphology and extension. Models with a detailed chemical treatment and with a comparison of the predicted chemical evolution with the observed metallicity of the target galaxy are still missing in the literature.

In two previous papers (Recchi et al. 2001, hereafter RMD; Recchi et al. 2002, hereafter R02), we explored the possibility of a star formation (SF) occurring in instantaneous burst(s) in a model galaxy resembling IZw18. The main results of these two papers are:

1. the abundances of heavy elements observed in IZw18 can be reproduced either with a single burst model, with an age of 31-35 Myr, depending on the model, or with two instantaneous bursts of star formation separated by a quiescent period of 300-500 Myr. The favoured age of the youngest generation of stars is 4-7 Myr, depending on the models. A second solution (with an age of several tens of Myr) is present, but it cannot be accepted since it does not reproduce the colors and the spectral energy distribution observed in IZw18;

2. a galactic wind develops in almost all the models, as a consequence of the energy supplied by $\mathrm{SNe}$ (of both type Ia and type II) and stellar winds. This wind carries away mostly the metals freshly produced during the burst;

3. SNeIa explode in a medium already heated and diluted by the previous activity of SNeII, thus they can hardly loose energy due to radiative processes. Owing to that, the galactic wind is mostly triggered by SNeIa and the metals produced by this kind of $\mathrm{SNe}$ (mainly iron-peak elements) are ejected more easily than the products of SNeII (mostly $\alpha$-elements). The $[\alpha / \mathrm{Fe}]$ ratios outside the galaxy are therefore lower than inside;

4. the cooling of metals in the gas has been found to be more efficient than expected and most of the freshly produced metals are already found in a cold phase (i.e. with temperatures below $2 \times 10^{4} \mathrm{~K}$ ) after 10-12 Myr (but see Sect. 5.1.2 for a critical discussion about this point). This can justify the so-called "instantaneous mixing" approximation, commonly assumed in chemical evolution models (Matteucci 1996, and references therein).

In this paper, we propose to relax the hypothesis of instantaneous bursts of star formation and analyze models with a more complex star formation regime, as suggested by fitting the observed Color-Magnitude Diagram (CMD) with synthetic ones (Aloisi et al. 1999; hereafter ATG). In Sect. 2 we summarize the current literature claims about the age and the past star formation history (SFH) of IZw18; in Sect. 3 we describe the model and the assumptions adopted in our simulations. In Sect. 4 we present our results. We discuss them in Sect. 5 and draw some conclusion in Sect. 6.

\section{The history of the star formation history determinations of IZw18}

IZw18 is a very blue galaxy $(U-B=-0.88$; van Zee et al. 1998 ), originally described by Zwicky (1966) as a double system of compact galaxies. Following studies have shown that these two components are two compact superclusters within the same galaxy, separated by 5.'8 (Papaderos et al. 2002). The two star forming regions are associated with two $\mathrm{H}_{\text {II }}$ regions: the NW and the SE region. Both regions are embedded in a low surface brightness envelope, extending to a radius of $\sim 1 \mathrm{kpc}$ (Dufour \& Hester 1990; Martin 1996) and a much more extended $\mathrm{H}_{\mathrm{I}}$ envelope, which has a size of several kpc and a total mass of $\sim 6 \times 10^{7} M_{\odot}$ (van Zee et al. 1998), but only $\sim 2 \times 10^{7} M_{\odot}$ of $\mathrm{H}_{\mathrm{I}}$ are associated with the optical part of the galaxy (Lequeux \& Viallefond 1980; van Zee et al. 1998). The total $\mathrm{H}_{\text {II }}$ mass is of the order of $3 \times 10^{6} M_{\odot}$ (Dufour \& Hester 1990). The optical part of the galaxy is often referred to as the Main Body. Hereafter we identify this region as the Galactic Region.

Historically, IZw18 has been considered as a very young galaxy, probably experiencing star formation for the first time (Searle \& Sargent 1972; Hunter \& Thronson 1995; Dufour et al. 1996). The presence of WR stars in the brighter (NW) component (Izotov et al. 1997a; Legrand et al. 1997; de Mello et al. 1998; Brown et al. 2002) indicate an ongoing star formation, or a star formation which has stopped only a few Myr ago.

The age of IZw18 also has been derived from a comparison of the morphology and the extension of the supershell with simple dynamical models of superbubble evolution (Martin 1996). The resulting age ranges between 15 and 27 Myr, depending on whether the star formation is continuous or occurs in an instantaneous burst. The chemical evolution models of 
Kunth et al. (1995) were able to reproduce the C, N, O and Fe abundances of IZw18 by assuming either a single or two very short bursts (with a duration of no longer than 10-20 Myr), separated by a quiescent period of $1 \mathrm{Gyr}$. The integrated Spectral Energy Distribution of IZw18 can be reproduced either with an instantaneous burst with an age of $\sim 3 \mathrm{Myr}$, or with a continuous burst lasting for $13 \mathrm{Myr}$ (Mas-Hesse \& Kunth 1999). Older stars are not required. Takeuchi et al. (2003) tried to reproduce the Infrared Spectral Energy Distribution observed in IZw18 by means of a dust production model, based on Hirashita et al. (2002) prescriptions. These authors predict an age range between 10 and $40 \mathrm{Myr}$ and do not invoke the presence of older stars.

However, the relatively high $\mathrm{C} / \mathrm{O}$ and $\mathrm{N} / \mathrm{O}$ abundance ratios seem to be justified only by assuming the presence of stars as old as a few hundred Myr (Dufour et al. 1988; Garnett et al. 1997) (note that the $C$ abundance in IZw18 has been recalculated by Izotov \& Thuan 1999 and seems to be in agreement with the $\mathrm{C} / \mathrm{O}$ observed in similar metal-poor BCDs). Also the remarkable global homogeneity of the chemical abundances in IZw18 led Vílchez \& Iglesias-Páramo (1998) to invoke the presence of an older stellar population, since otherwise the distribution of metals would be more patchy. A breakthrough in the age and SFH determination of IZw18 has come with the work of ATG. By means of a careful analysis of Hubble Space Telescope WFPC2 archival data, these authors were able to detect post-main sequence stars, identified as Asymptotic Giant Branch (AGB) stars, with an age of several $10^{8} \mathrm{yr}$, as derived from the comparison of the observed CMD with synthetic ones. This result has been confirmed by Östlin (2000), who analyzed the CMD in the near infrared, revealing the presence of stars as old as a few Gyr. The relative importance of this older stellar population compared to the younger one is however still matter of debate. The number of red point sources detected both in the optical and in the NIR ranges by HST (ATG; Östlin 2000) is not more than a dozen and the Spectral Energy Distribution of IZw18 is reproduced without the need for stars older than 13 Myr (Mas-Hesse \& Kunth 1999). Kunth \& Östlin (2000) proposed that the low surface brightness component of IZw18 can be due to a disc of stars as old as 1-5 Gyr, but this finding has been debated by Papaderos et al. (2002). These authors found that the low surface brightness envelope of the galaxy is not due to an old disc component, but rather to an extended ionized gas emission.

An extreme model has been proposed by Legrand (2000) and Legrand et al. (2000). It has been proposed that the chemical and photometric properties of IZw18 can be reproduced assuming a very low star formation rate $\left(S F R=10^{-4} M_{\odot} \mathrm{yr}^{-1}\right)$, lasting for a Hubble time, with a recent burst of star formation superimposed. More recently, Hunt et al. (2003), by fitting colors of different regions of IZw18 with evolutionary synthesis models, derived an age of at most $500 \mathrm{Myr}$ for the oldest stellar population and at most $15 \mathrm{Myr}$ for the youngest one in the main body. Stars older than $500 \mathrm{Myr}$ are not detected. If any, they would contribute by no more than $22 \%$ in mass ( $4 \%$ in $J$ light).

In conclusion, the idea that IZw18 is experiencing the very first burst of star formation is definitively ruled out. On the other hand, the recent literature seems to agree on the fact that very old stars (stars older than $0.5-1 \mathrm{Gyr}$ ), if any, do not produce a significant contribution to the light and the metal budget of IZw18. We therefore adopt hereafter the SFH inferred by ATG.

\section{The model}

We simulate a galaxy model resembling IZw18 by means of a 2-D hydrocode in cylindrical coordinates, coupled with detailed chemical yields originating from SNeII, SNeIa and winds from Intermediate-Mass Stars (IMS). The SNeIa rate is calculated according to the so-called Single-Degenerate scenario (namely $\mathrm{C}-\mathrm{O}$ white dwarfs in binary systems that explode after reaching the Chandrasekhar mass because of mass transfer from a red giant companion). This rate can be calculated analytically for an instantaneous burst of star formation (Greggio \& Renzini 1983; Matteucci \& Recchi 2001) and it peaks after a few tens of Myr. For massive stars we adopt the nucleosynthetic yields of Woosley \& Weaver (1995), whereas for the IMS we consider both the yields of Renzini \& Voli (1981, hereafter RV81) and the ones of van den Hoek \& Groenewegen (1997, hereafter VG97). For RV81 we chose the set of yields with $Z=0.004$ and $\alpha=1.5$, whereas among the calculations of VG97 we chose the yields with $Z=0.001$ and $\eta_{\mathrm{AGB}}=4$. In Sect. 4.4 we analyze two models in which new yields of Meynet \& Maeder (2002, hereafter MM02), for both massive stars and IMS, are implemented. The details about the code and the computation of the chemical enrichment can be found in RMD and R02.

According to the results of ATG, we adopt a star formation constant for $270 \mathrm{Myr}$ (with $S F R=6 \times 10^{-3} M_{\odot} \mathrm{y}^{-1}$ ), a quiescent period of $10 \mathrm{Myr}$ and a second, more vigorous burst $(S F R=$ $3 \times 10^{-2} M_{\odot} \mathrm{y}^{-1}$ ), lasting for $5 \mathrm{Myr}$. Models with a weaker SFR in the first, long-lasting episode of SF, are analyzed in Sect. 4.3.

We calculate the mass of stars formed between $t$ and $t+\Delta t$, where $\Delta t=10^{5} \mathrm{yr}$. This Stellar Population (SP) is treated as a starburst. We thus, according to the adopted IMF slope and chemical yields, calculate the mass, energy and metal released by each SP. The metals released by each SP will vary according to the metallicity of the stars. The rate of restitution of each chemical element $l$ into the ISM from SNeII is calculated as follows:

$$
\begin{aligned}
\dot{m}_{\mathrm{II}}^{l}(t)= & \sum_{i} \phi[m(t-i \cdot \Delta t)]\left[\Delta m(t-i \cdot \Delta t) X^{l}(i)\right. \\
& \left.+m(t-i \cdot \Delta t) P^{l}(m)\right]\left|\frac{\mathrm{d} M}{\mathrm{~d} t}\right|_{t-i \cdot \Delta t}
\end{aligned}
$$

where the sum is extended to all the SP young enough to contain live stars more massive than $8 M_{\odot}$. We consider in this equation both the contribution of newly synthesized metals $\left(m(t-i \cdot \Delta t) P^{l}(m)\right)$, and of metals released by the stars but unmodified by nucleosynthetic processes $\left(\Delta m(t-i \cdot \Delta t) X^{l}(i)\right)$. In a similar way we calculate the mass loss rate from SNeIa and intermediate-mass stars. Analogously to what we have done in RMD and R02, we consider here the evolution, in space and time, of 8 chemical elements of particular astrophysical relevance, namely $\mathrm{H}, \mathrm{He}, \mathrm{C}, \mathrm{N}, \mathrm{O}, \mathrm{Mg}, \mathrm{Si}, \mathrm{Fe}$. 
We assume a SF occurring at the center of the system and consequently we inject energy and metals uniformly in this central region. This picture is simplistic, since star formation occurs mostly in star clusters (Meurer et al. 1995; Fall 2004), which can be particularly large (super star clusters) in dwarf irregular galaxies (Larsen 2002). However, due to the relatively low resolution of our simulations and to the assumption of the symmetry along the polar axis, is it not easy to simulate the sites of star formation and release of energy and metals in a more accurate way. We have to take into consideration this limitation, when comparing the results of our simulation with IZw18 (see also Sect. 5.1.1).

Our code is able to calculate at each time-step the metallicity of the star forming region, thus varying the $X^{l}(i)$ term in Eq. (1) in a self-consistent way. However, our simulation cannot spatially resolve molecular clouds (with sub-parsec dimensions, see e.g. Matsumoto \& Hanawa 2003). The calculation of the metallicity of each stellar population would be obtained just by averaging the metal content of the cold gas in the star forming region, which is a rather poor approximation. Moreover, the superbubble created by the ongoing star formation soon fills the whole star forming region. The gas present in this region is therefore diluted, hot (thus unable to form stars) and enriched in metals. For this reason, it is hard to reconcile the hypothesis of a varying metallicity of the stellar populations with the centrally concentrated star formation. We therefore assume, in this study, a constant metallicity of $1 / 100 Z_{\odot}$, according to the findings of ATG. This is a simplistic, but not unrealistic, assumption.

Following the assumptions of RMD, we adopt a low thermalization efficiency for SNeII $\left(\eta_{I I}=0.03\right.$; see details in RMD), namely only $3 \%$ of the explosion energy of $\mathrm{SNe}$ is available to thermalize the ISM, whereas the rest is radiated away during the expansion of the Supernova Remnant. Actually, the first $\mathrm{SNe}$ heat and dilute the gas, thus in principle the thermalization efficiency should increase with time. However, SNeII arise from short-living stars (lifetimes $\sim 3-30 \mathrm{Myr}$ ). These stars are born in molecular clouds, thus always in a dense and cold environment, irrespective of the energy released by the other $\mathrm{SNe}$ before. Thus, if SNeII explode, they have to explode in a medium relatively cold and dense, due to the short lifetime of massive stars. If the medium is hot and diluted, stars cannot form and, of course, SNeII cannot explode. On the other hand, SNeIa arise from binary systems with lifetimes spanning from some tens of Myr to several Gyr. The progenitor stars are thus born in a cold environment, but by the time at which the explosion occurs, the thermodynamical conditions of the circumstellar medium can be drastically different. We can therefore safely assume a very high thermalization efficiency for SNeIa (namely $\eta_{I a}=1$ ). The effect of a lower SNeIa thermalization efficiency is analyzed in Sect. 4.3. Note that the low thermalization efficiency for SNeII, disputed in some papers (Strickland \& Stevens 2000; Summers et al. 2003) has been confirmed, at least in the first $\sim 16$ Myr of burst activity, by Melioli \& de Gouveia dal Pino (2004).

Both SNeIa and SNeII are assumed to have an explosion energy of $10^{51} \mathrm{erg}$. Some recent papers show a possible dependence of the explosion energy on the progenitor mass, from both a theoretical (Fryer 1999; Fryer \& Kalogera 2001) and an observational (Nomoto et al. 2003) point of view. However these results contradict each other, thus no firm conclusion can be drawn about this point and the assumption of a constant explosion energy seems to be the safest one.

\section{Results}

\subsection{The standard model}

\subsubsection{Dynamical results}

The initial conditions chosen for the standard model (SR2) are almost identical to the starting model assumed in RMD and R02 papers, namely a rotating gaseous component in hydrostatic isothermal equilibrium with the galactic potential (sum of a spherical dark halo and a flattened stellar component) and the centrifugal force. The dark matter halo extends over $10 \mathrm{kpc}$, whereas the stellar component is distributed in an oblate structure with dimensions $\sim 1 \mathrm{kpc} \times 700 \mathrm{pc}$, similar to the distribution of stars observed in IZw18 (Dufour \& Hester 1990; Papaderos et al. 2002). This model assumes a Salpeter IMF ( $x=1.35$ ) between 0.1 and $40 M_{\odot}$.

In Fig. 1 is shown a snapshot of the evolution of this model during the first $\sim 200$ Myr. The energy released in the gas by means of stellar winds and $\mathrm{SNe}$ is enough to unbind the gas and already create a galactic wind after $\sim 100 \mathrm{Myr}$. The mass of stars formed after $100 \mathrm{Myr}$ is $6 \times 10^{5} M_{\odot}$, similar to the mass of the burst assumed in the standard model of RMD. Given the similar assumptions about the thermalization efficiency, the total energy released by SNeII is thus the same $\left(\sim 2 \times 10^{53} \mathrm{erg}\right)$. This energy is released on a time-scale three times longer than in the case of an instantaneous burst of star formation, thus the luminosity of the burst is three times lower $\left(L_{w} \sim 6.5 \times 10^{38} \mathrm{erg} \mathrm{s}^{-1}\right)$. The SNeIa contribution however already becomes non-negligible after a few tens of Myr and after 100 Myr the luminosity produced by SNeIa is comparable with the luminosity by SNeII. Considering also the contribution of stellar winds from intermediate-mass stars, a jet-like structure is expected from simple energetical considerations (see RMD).

A magnification of the second panel of Fig. 1 is shown in Fig 2. In this figure we can observe the classical two-shock configuration (Weaver et al. 1977): a forward shock is propagating through the ISM. Due to the steep density gradient, the shocked ISM shell accelerates, promoting Rayleygh-Taylor instabilities and fragmenting into cloudlets and filaments visible in Fig. 2. The reverse shock thermalizes the freely expanding wind, heating it up to temperatures of the order of a few $10^{6} \mathrm{~K}$. The reverse shock is approximately spherical, since the short sound-crossing time (of the order of $1 \mathrm{Myr}$ ) keeps the pressure almost uniform in the inner region. The extension along the disc is $\sim 550 \mathrm{pc}$. This is marginally consistent with the maximal allowed value $0.72 H_{\text {eff }}\left(L_{w} / L_{b}\right)^{1 / 6} \sim 420 \mathrm{pc}$, where $H_{\text {eff }}$ is the effective scale-length of the ISM distribution in the polar direction (Koo \& McKee 1992). This is also a slightly larger value compared with the maximal extension in the symmetry plane reached by the model with an instantaneous burst of star formation (see RMD, their Fig. 5), although the burst luminosity is comparable. This is apparently at variance with the 


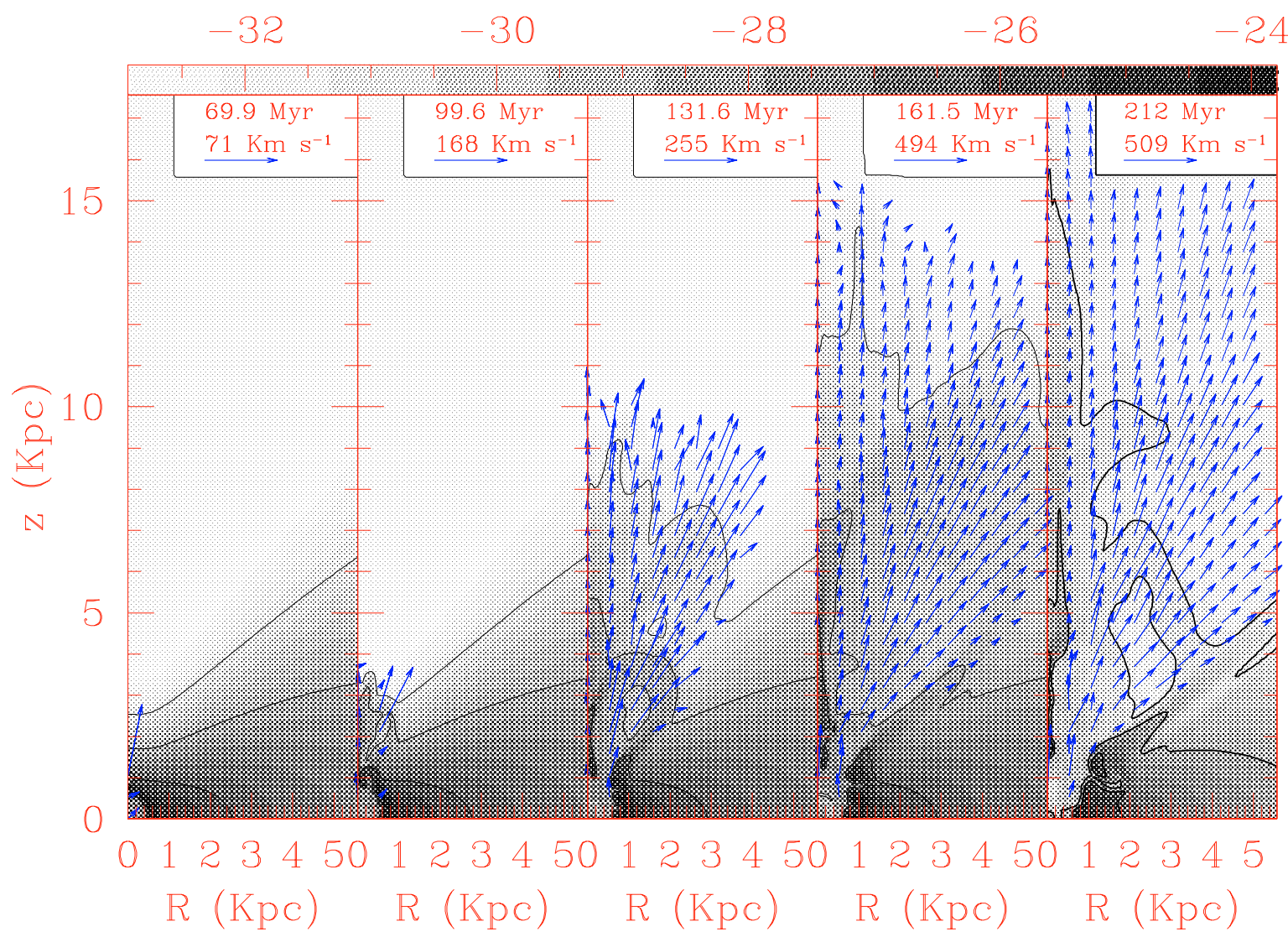

Fig. 1. Density contours and velocity fields for model SR2 at different epochs (evolutionary times are labelled in the box on top of each panel). The logarithmic density scale is given in the strip on top of the figure. In order to avoid confusion, velocities with values lower than $1 / 10$ of the maximum value (indicated for each panel in the upper right box) are not drawn. This is true also for Figs. 2 and 6.

findings of Strickland \& Stevens (2000), who show a slightly slower superbubble evolution when the energy and the mass injection are more gradual. A more careful analysis of their Fig. 2 shows that the energy injection rate for the "continuous" model (named CSF by the authors) is comparable to the luminosity of the starburst model only at $t \sim 7.5 \mathrm{Myr}$ (the evolutionary time adopted for the comparison), whereas for $t<7.5 \mathrm{Myr}$ the luminosity of the CSF model is significantly lower, thus the total energy injected by $\mathrm{SNe}$ in this model is much below the energy released by the "starburst" model, at variance with our results.

At the end of the simulation (after $\sim 315 \mathrm{Myr}$ ), almost half of the gas initially present inside the galactic region is lost through the galactic wind. Only $\sim 9 \times 10^{6} M_{\odot}$ of gas are kept bound. This is a factor of $\sim 2$ lower than the neutral gas content of the main body of IZw18, thus this model cannot be the best one in order to reproduce the morphological properties of IZw18, but nevertheless it allows us to compare the effect of different star formation histories (single, instantaneous burst of SF; double burst of SF; continuous SF) starting from a similar ISM distribution.

\subsubsection{Chemical evolution}

The evolution of the $\mathrm{C}, \mathrm{N}, \mathrm{O}$ abundances in the cold medium (i.e. integrating over the grid points in which the temperature is below $2 \times 10^{4} \mathrm{~K}$ ) is shown in Fig. 3 (solid line). This evolution is compared with the evolution of similar models (same initial gaseous distribution, same IMF, same nucleosynthetic prescriptions, namely the RV81 ones) but with different histories of star formation. The dotted line represents the standard single burst model of star formation (already shown in RMD and labelled as model M1B therein). The dashed line represents a model with two instantaneous bursts of star formation, already shown in R02 (the model called M300R).

These models show different chemical evolution patterns and it is worth comparing them to understand the effect of the star formation history on the chemical evolution of galaxies. While carbon and oxygen abundances are reproduced at the end of the simulation of the continuous burst model, the nitrogen is well above $(\sim 0.5 \mathrm{dex})$ the values observed in IZw18. This is due to the fact that the last burst of star formation is only 5 times more intense than the average SFR and lasts for only $5 \mathrm{Myr}$. The oxygen produced is not enough to compensate for the nitrogen coming from the IMS. Moreover, most of the metals produced in the second episode of star formation do not have time to cool below $2 \times 10^{4} \mathrm{~K}$, since they are immediately channelled along the galactic funnel, so they do not contribute to the chemical enrichment of the galaxy. At variance with the double-burst model (dashed line), the signature of the onset of the second episode of SF is not recognizable. In order to reproduce the $\mathrm{N} / \mathrm{O}$ observed in IZw18, we have to reduce the $\mathrm{N}$ yields calculated by RV81 by a factor of 3 (long-dashed line in 




Fig. 2. Density contours and velocity fields for model SR2 after $\sim 100 \mathrm{Myr}$ (magnification of the second panel of Fig. 1).

Fig. 3). If we instead adopt the yields of VG97, a reduction of $\mathrm{N}$ yields by a factor of $\sim 2$ is enough to fit the observed values of IZw18.

Quite surprisingly, the nitrogen produced by the continuous burst model during the first, long-lasting episode of star formation, is well below the value reached by the instantaneous burst models. This is only marginally due to the star formation history considered in this model, and is mostly due to the effect of the galactic wind. We can calculate the ejection efficiencies $\eta_{l}$ by simply dividing the mass of the gas in the form of the single chemical element $l$ that has left the galaxy $\left(M_{\text {out }}^{l}\right)$ by the total mass of the chemical species $l$ produced during the episode of star formation, namely:

$\eta_{l}=\frac{M_{\mathrm{out}}^{l}}{M_{\mathrm{in}}^{l}+M_{\mathrm{out}}^{l}}$.

The evolution of the ejection efficiencies of $\mathrm{C}, \mathrm{N}, \mathrm{O}$ and $\mathrm{Fe}$ for the model SR2 are shown in Fig. 4. These four elements are chosen because they are synthesized by different kinds of stars, on different time-scales. Oxygen is mostly produced by

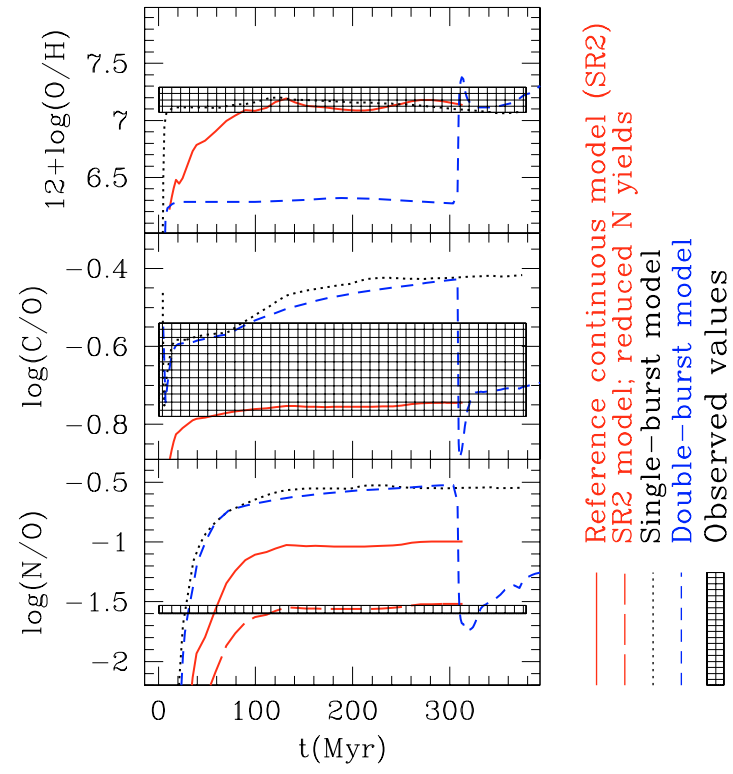

Fig. 3. Evolution of $\mathrm{O}, \mathrm{C}, \mathrm{N}$ for the reference continuous burst model (solid line). This model is compared with another model with an instantaneous burst of star formation (dotted line) and a model with two bursts of star formation separated by a quiescent period of $300 \mathrm{Myr}$ (dashed line). The long-dashed line is a model in which the $\mathrm{N}$ yields are reduced by a factor of 3 . The superimposed dashed areas represent the observative values, with relative error-bars, found in literature for IZw18. This is true also for Figs. 7, 8 and 10.

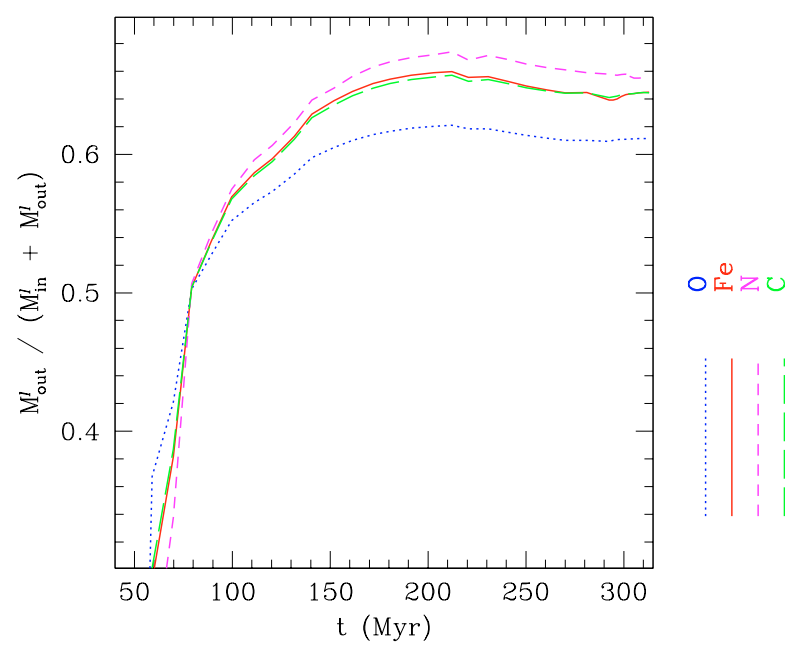

Fig. 4. Ejection efficiencies $\left(\eta_{l}=M_{\text {out }}^{l} /\left(M_{\text {in }}^{l}+M_{\text {out }}^{l}\right)\right)$ as a function of time for the model SR2.

quiescent burning in massive stars and ejected by type SNeII, on a very short time-scale. Nitrogen can either be produced in a "secondary" way, namely from the $\mathrm{C}$ and $\mathrm{O}$ already present in the star at birth, or in a "primary" way, namely starting from the $\mathrm{C}$ and $\mathrm{O}$ freshly produced in the star and dredged up during the thermal pulsing phase when the star is on the AGB phase (RV81). The secondary production of nitrogen depends on the metallicity; therefore in metal-poor galaxies the primary production in intermediate-mass stars is the dominant process. It has also been suggested that a significant fraction of $\mathrm{N}$ might be produced in a primary way in massive stars 


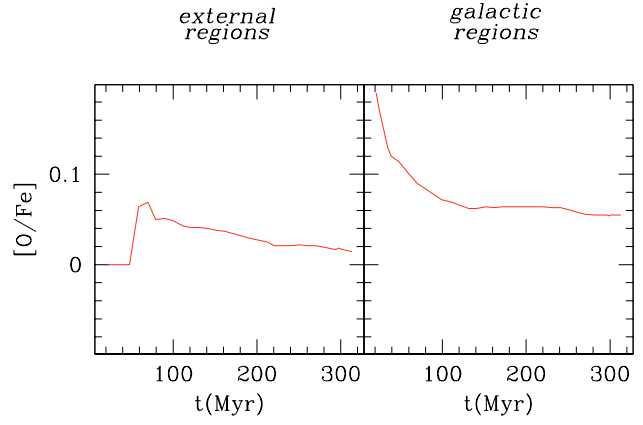

Fig. 5. $[\mathrm{O} / \mathrm{Fe}]$ vs. time for the model SR2.

(Matteucci 1986; Izotov \& Thuan 1999), but the N necessary to reproduce the N/O values observed in metal-poor dwarf galaxies is well above the predictions of nucleosynthetic papers (see also the discussion in R02). The carbon origin has also been debated, since some authors (Carigi 2000; Henry et al. 2000) believe that $\mathrm{C}$ is mainly produced in massive stars. Chiappini et al. (2003a) suggested instead that $\mathrm{C}$ comes mainly from low- and intermediate-mass stars, although the contribution of massive stars is non-negligible. Finally, Fe comes mainly from SNeIa, although SNeII produce around one third of the observed $\mathrm{Fe}$ (Matteucci \& Greggio 1986).

As we can see from Fig. 4, the ejection efficiencies become very large on a time-scale shorter than $\sim 100 \mathrm{Myr}$. At the beginning, oxygen is ejected more efficiently than the other elements. This is due to the fact that the first chemical elements that experience the effect of the galactic wind are the SNeII products. At later times, however, the ejection efficiencies of the elements produced on longer time-scales become comparable and then slightly larger than the ejection efficiencies of $\alpha$-elements. This is due to the fact that SNeII make the strong effort of breakingout and carving the galactic funnel. When the bulk of SNeIa and intermediate-mass stars release their nucleosynthetic products into the ISM, the tunnel has already been carved and it is easy for chemical elements produced on longer time-scales to be channeled and ejected out of the galaxy. This effect has already been found and analyzed by RMD and R02. The lower $\mathrm{N} / \mathrm{O}$ in the continuous model, observed in Fig. 3, is due in part to the very large ejection efficiency of $\mathrm{N}$, in part to the fact that SNeII and SNeIa continuously pump energy into the system, thus the amount of $\mathrm{N}$ with a temperature larger than $2 \times 10^{4} \mathrm{~K}$ (thus not considered in this plot) is larger than in the models with instantaneous bursts of star formation.

The $[\mathrm{O} / \mathrm{Fe}]$ ratios outside and inside the galaxy are shown in Fig. 5. Due to the different ejection efficiencies of $\mathrm{O}$ and $\mathrm{Fe}$ seen in Fig. 4, there is a small (less than 0.1 dex) but visible difference in the $[\alpha / \mathrm{Fe}]$ ratios outside and inside the galaxy. This figure also shows a maximum in the $[\mathrm{O} / \mathrm{Fe}]$ in the gas flowing outside the galaxy at $\sim 70-80 \mathrm{Myr}$, namely at the moment of the onset of the galactic wind. This means that the wind is initially enriched in $\alpha$-elements, as seen in the outflow of the starburst galaxy NGC 1569 by Martin et al. (2002). The bulk of Fe in the outflow comes afterwards, due to the minor work required to eject iron-rich gas when the funnel has already been carved.

The $[\mathrm{O} / \mathrm{Fe}]$ shown in Fig. 5 is still normalized to the Anders \& Grevesse (1989) solar values, although it is now well
Table 1. Parameters for the continuous burst models.

\begin{tabular}{cccc}
\hline \hline Model & Gas mass $\left(M_{\odot}\right)$ & $x$ (IMF slope) & IMS yields \\
\hline SR2 & $1.7 \times 10^{7}$ & 1.35 & RV81 \\
SR3 & $3.0 \times 10^{7}$ & 1.35 & RV81 \\
SV3 & $3.0 \times 10^{7}$ & 1.35 & VG97 \\
KR2 & $1.7 \times 10^{7}$ & 1.7 & RV81 \\
FV4 & $4.0 \times 10^{7}$ & 0.5 & VG97 \\
\hline
\end{tabular}

established that these authors overestimated the solar oxygen composition (Holweger 2001). We keep this value since, due to the difficulties in the estimation of the iron content in IZw18 (see e.g., Izotov et al. 1997b; Levshakov et al. 2001) and the uncertainties in the calculation of Fe yields (Timmes et al. 1995) we believe it is meaningless to directly compare the results of our simulations with the $\mathrm{Fe}$ and $[\alpha / \mathrm{Fe}]$ observed in IZw18 and thus we concentrate on the comparison between the $[\alpha / \mathrm{Fe}]$ inside and outside the galaxy, since it can give us some hints about the chemical enrichment of the intracluster medium (ICM).

\subsection{Exploring the parameter space}

As stated in Sect. 4.1.1, model SR2 is not satisfactory to reproduce the morphological properties of IZw18, since the final gas content is lower than the observed one. Moreover, it is possible to reproduce the abundances of $\mathrm{C}, \mathrm{N}$ and $\mathrm{O}$ at the same time only by reducing the $\mathrm{N}$ yields coming from IMS (see Fig. 3). It is thus worth changing the initial mass of gas present inside the galaxy, the adopted set of nucleosynthetic yields and the IMF slope (Salpeter in the standard model) and explore the parameter space to find the best model able to fit the chemical and morphological properties of IZw18. We test here different IMF slopes, different sets of yields from IMS and different masses of gas at the beginning of the simulation. In particular, a flatter $(x=0.5)$ IMF slope has been suggested by ATG to reproduce the observed CMD in IZw18. A steeper $(x=1.7)$ IMF has been instead suggested by Scalo (1986) and Kroupa et al. (1990) for massive stars in the Galaxy (see also Kroupa 2002, for a review of IMF determinations). The model parameters are shown in Table 1. The identification of a particular model is made through the notation $\mathrm{XYN}$, where $\mathrm{X}$ refers to the adopted IMF slope (S: Salpeter; K: Kroupa; F: flat), Y individuates the adopted IMS yields (R: Renzini \& Voli 1981; V: van den Hoek $\&$ Groenewegen 1997) and $\mathrm{N}$ is approximately the gas mass (in $10^{7} M_{\odot}$ ) in the galactic region at the beginning of the simulation. Therefore, the reference SR2 represents a model with Salpeter IMF, RV81 yields and approximately $2 \times 10^{7} M_{\odot}$ of Hi gas.

\subsubsection{Dynamical evolution}

As mentioned in Sect. 4.1.1, in the reference model (SR2) a galactic wind already occurs after $\sim 100 \mathrm{Myr}$ (see Fig. 2). It is useful to compare the evolutionary status of the models of Table 1 after $\sim 100 \mathrm{Myr}$, to study how initial gas distribution and the IMF affect the evolution of the galaxy. In Fig 6 we show a comparison of density contours for models SR2, SV3, 


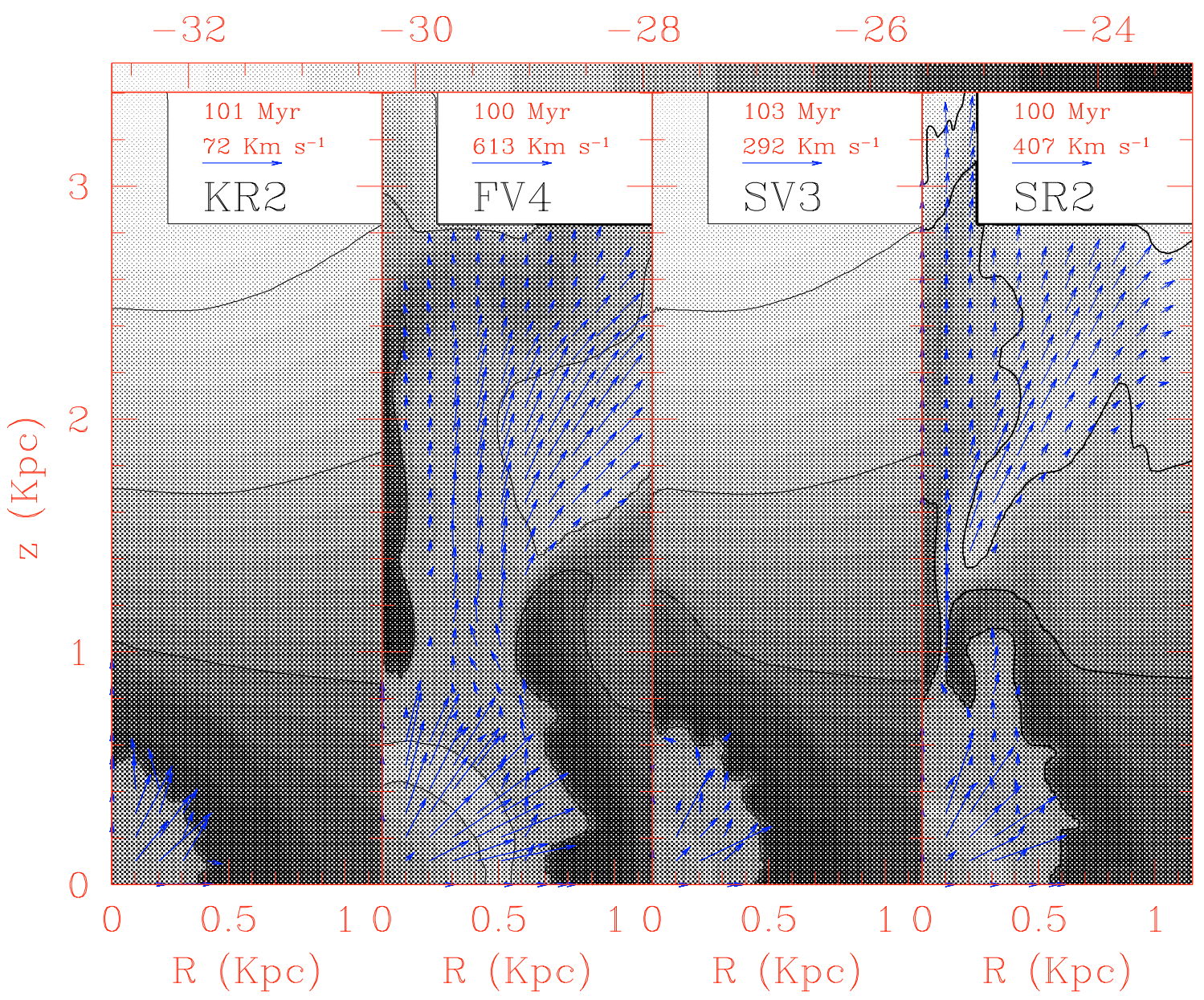

Fig. 6. Density contours and velocity fields for models SR2, SV3, KR2, FV4 (parameters of these models in Table 1) at $\sim 100$ Myr.

KR2, FV4. Model SR3 is not shown since, from a dynamical point of view, it is almost indistinguishable from model SV3.

As evident in this figure, only models SR2 (fourth panel) and FV4 (second panel) already develop a galactic wind after 100 Myr. Model FV4, in spite of the very high gas content at the beginning of the simulation, ejects more than $80 \%$ of the initial mass through the very powerful galactic wind. The simulation lasts for $\sim 330 \mathrm{Myr}$ and the final gas content is $\sim 7.6 \times 10^{6} M_{\odot}$, much below the observed value. The flat IMF strongly favors the occurrence of type II SNe. The ratio between the number of SNeII in the FV4 (with a $x=0.5$ IMF slope) model and the one in the model with a Salpeter IMF is simply given by:

$\frac{N_{\mathrm{SNII}}^{\mathrm{FV} 4}}{N_{\mathrm{SNII}}^{\mathrm{SR} 2}}=\frac{\int_{8}^{40} m^{-1.5} \mathrm{~d} m}{\int_{8}^{40} m^{-2.35} \mathrm{~d} m} \simeq 10$

Since we assumed in any models the same prescriptions for the thermalization efficiencies, the model FV4 produces $\sim 10$ times more energy by SNeII than the model SR2. A difference of a factor of $\sim 4$ is also present in the total energy provided by SNeIa. This difference is approximately proportional to the ratio between the number of stars in the range $[3,16] M_{\odot}$. The total energy provided by SNe in the model FV4 is thus a factor of $\sim 7$ larger than the energy provided in the model SR2. The binding energy of the gas is instead only a factor of $\sim 2$ larger and this explains the remarkable difference in the wind efficiency in the two models.

Model KR2 (first panel in Fig. 6), with a steeper ( $x=1.7$ ) IMF slope, produces much less energy through $\mathrm{SNe}$ (more than a factor of 2 lower), thus the development of the galactic wind is much slower. Only $\sim 10 \%$ of the gas initially present in the galaxy is lost through the galactic wind, thus the gas content of this model is consistent with the observations. Finally, model SV3 (third panel in Fig. 6) develops a weak galactic wind at around $170 \mathrm{Myr}$. The final gaseous mass is $\sim 2.2 \times 10^{7} M_{\odot}$, thus marginally consistent with the gas content observed in IZw18.

\subsubsection{Chemical evolution}

The resulting evolution of $\mathrm{O}, \mathrm{C} / \mathrm{O}, \mathrm{N} / \mathrm{O}$ for the models described in Table 1 is shown in Fig. 7. As can be seen from the figure, none of these models can reproduce the observed $\mathrm{N} / \mathrm{O}$ ratio in a satisfactory way. Only the model FV4 (dotdashed lines in Fig. 7), with a flatter $(x=0.5)$ IMF slope can account for the low $\mathrm{N}$ content (indeed even lower than the observed value), but this model overproduces $\mathrm{O}$ (and, consequently, the predicted $\mathrm{C} / \mathrm{O}$ is below the observed values). Moreover, as described in Sect. 4.2.1, this model is unable to reproduce the mass content of IZw18. 


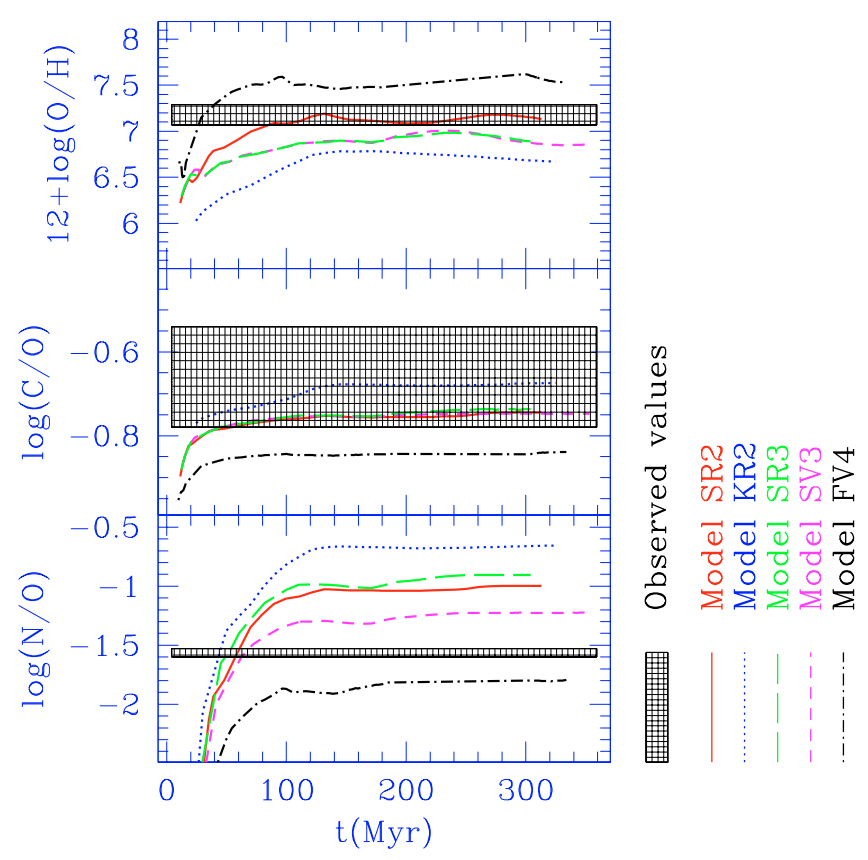

Fig. 7. Evolution of $\mathrm{O}, \mathrm{C}, \mathrm{N}$ for the continuous burst models. The parameters adopted for each model are described in Table 1.

Model SR2 (solid lines in Fig. 7) is the reference model and it has been already described in Sect. 4.1.2 and shown in Fig. 3. Compared to model SR2, model SR3 (long-dashed lines in Fig. 7) contains a dilution of metals produced by the ongoing star formation in a larger amount of gas, thus resulting in a lower oxygen abundance. This is also true for the model SV3 (dashed lines in Fig. 7). The main difference between model SR3 and SV3 is on the N production. In fact, $\mathrm{O}$ is mostly produced in massive stars, and a change in the adopted IMS yields does not influence the $\mathrm{O}$ production. On the other hand, $\mathrm{C}$ is produced both by massive stars and by IMS, with a significant contribution from masses between 1 and $3 M_{\odot}$ (see Chiappini et al. 2003a). There is some difference in the C production between RV81 and VG97 nucleosynthetic yields, but the difference arises more clearly after the lifetime of a $3 M_{\odot}$ star, larger than the considered evolutionary time in our simulations.

Model KR2 (dotted lines in Fig. 7), with a steeper $(x=1.7)$ IMF slope, is strongly biased towards low mass stars, therefore it overproduces $\mathrm{N}$ and underproduces $\mathrm{O}$. Due to the fact that it underproduces the $\mathrm{C}$ coming from massive stars, as well, it provides a good fit of the $\mathrm{C} / \mathrm{O}$ abundance ratio. Such a very steep IMF in BCD galaxies can therefore be ruled out. The best IMF slope in order to reproduce the characteristics of IZw18 seems therefore to be the Salpeter (1955) one or flatter (but steeper than $x=0.5$ ).

\subsection{Models with different star formation rates and different SNela thermalization efficiencies}

In order to explore the effect of the variations in the SFH, we ran two models (labelled SV3b and SV3c), similar to SV3 model, but with reduced SFR. The SFR during the first,
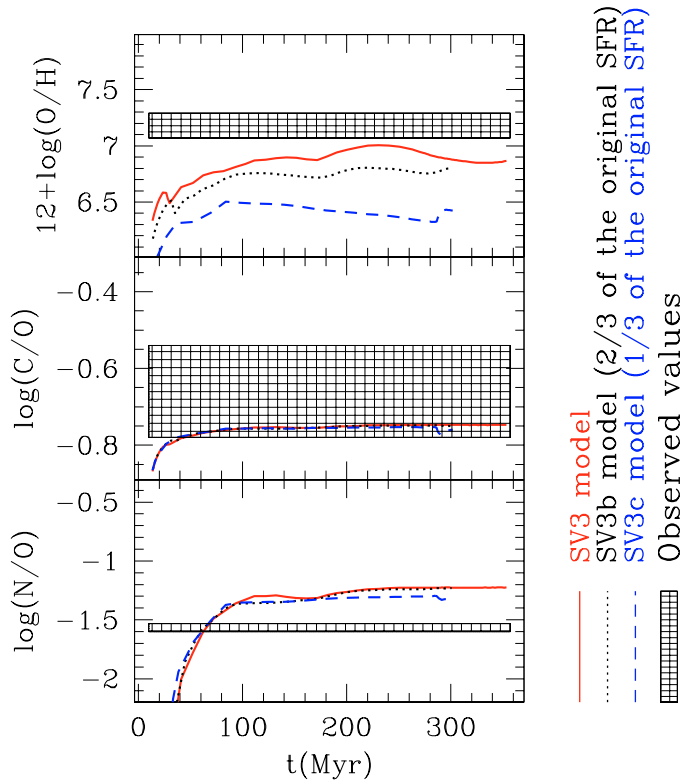

Fig. 8. Evolution of O, C, N for the model SV3 (solid line) and for models with reduced SFR. Dotted line: model SV3b (SFR during the long-lasting burst $4 \times 10^{-3} M_{\odot} \mathrm{y}^{-1}$ ). Dashed line: model SV3c (SFR during the long-lasting burst $2 \times 10^{-3} M_{\odot} \mathrm{y}^{-1}$ ).

long-lasting episode of SF is $4 \times 10^{-3} M_{\odot} \mathrm{y}^{-1}$ (2/3 of the original value) for the model SV3b and $2 \times 10^{-3} M_{\odot} \mathrm{y}^{-1}(1 / 3$ of the original value) for the model SV3c. The SFR of the second, more recent burst of SF is unmodified.

The injected energy in these two models is strongly reduced, therefore the fraction of gas able to escape the galaxy is small. At the end of the simulation, the amount of gas still present in the galactic region is $\sim 2.8 \times 10^{7} M_{\odot}$ for model SV $3 b$ and $\sim 2.9 \times 10^{7} M_{\odot}$ for model SV3c.

The evolution of $\mathrm{C}, \mathrm{N}, \mathrm{O}$ for these 3 models is shown in Fig. 8. As expected, variations in the SFR do not appreciably change the $\mathrm{C} / \mathrm{O}$ and $\mathrm{N} / \mathrm{O}$ ratios, whereas the global production of oxygen is reduced. It is also worth noticing that, after the onset of the second episode of SF, a bump in the evolution of $\mathrm{O} / \mathrm{H}$ in the model SV3c arises. Such a bump is not observed in the model SV3 and is only barely visible in the model SV3b. As pointed out also in Sect. 4.1.2, in the models with large SFR, most of the metals produced in the second episode of SF are immediately channelled along the galactic wind and do not have the chance to cool below $2 \times 10^{4} \mathrm{~K}$, therefore the onset of the second episode of SF is not observed in the chemical evolution tracks. Moreover, the mass in stars formed in the second episode of SF is $\sim 8 \%$ of the total stellar mass of the galaxy for models with standard SFR. This fraction increases up to $\sim 12 \%$ for model SV3b and $\sim 22 \%$ for model SV3c. The metal production during the second episode of SF for these models therefore has a stronger impact on the global metal budget of the galaxy.

We also ran a model, similar to SV3, but with reduced thermalization efficiency for SNeIa $\left(\eta_{\text {Ia }}=0.1\right.$ instead of 1$)$. In this model the amount of gas kept bound in the galactic region at the end of the simulation is $\sim 2.8 \times 10^{7} M_{\odot}$, significantly more than the final gaseous mass in the model SV3 $\left(\sim 2.2 \times 10^{7} M_{\odot}\right)$. The $\mathrm{C}, \mathrm{N}, \mathrm{O}$ abundances at the end of the simulations for this 
model are slightly larger than in the SV3 model, the difference being of the order of $\sim 0.1 \mathrm{dex}$. This difference arises from the fact that more metals are kept bound inside the galactic region. Moreover, since less energy per unit mass is provided by the starburst, the fraction of cold metals is larger.

\subsection{Models with Meynet \& Maeder (2002) yields}

There are indications (Chiappini et al. 2003a) that the sets of yields of VG97 may overestimate the amount of nitrogen produced by intermediate mass stars. Recent models of stellar evolution with rotation (MM02) predict less $\mathrm{N}$ in IMS. MM02 yields were obtained from self-consistent complete stellar models. $\mathrm{N}$ is mainly produced in a primary way through rotational diffusion of $\mathrm{C}$ in the $\mathrm{H}$ burning shell. We emphasize here that these models do not take into consideration later phases of the stellar evolution (in particular the third dredgeup and the hot-bottom burning phase), and may underestimate the amount of primary nitrogen. However, the yields of VG97 and RV81 are obtained by means of synthetic AGB models, in which the production of $\mathrm{N}$ and $\mathrm{C}$, in particular during the hot-bottom burning phase, is parametrized following some "ad hoc" analytical relations, since none of the available stellar models adequately describe this phase of the stellar evolution. Hence, none of the available models in the literature seems to contain all the physics needed to reproduce the primary nitrogen production. Is it therefore interesting to test different sets of nucleosynthetic yields in order to put constraints on the primary production of $\mathrm{N}$ in IMS.

A comparison of the $\mathrm{N}$ yields in IMS coming from different authors is shown in Fig. 9. MM02 presented two sets of yields: the first one with a very low $\left(Z=10^{-5}\right)$ metallicity and the second one with a metallicity of $Z=0.004$. In the case of models with $Z=10^{-5}$, MM02 do obtain the third dredge-up, in the sense that the products of $\mathrm{H}$ and He burning reach the surface of the star. As can be seen in this figure, the predicted production of N in IMS is much below the values tabulated by RV81 and VG97. Only at very low initial masses VG97 yields are below the MM02 results, but this mass range $\left([1,3] M_{\odot}\right)$ produces a negligible amount of N. MM02 predict instead a (slightly) larger amount of primary $\mathrm{N}$ coming from massive stars, compared to the results of Woosley \& Weaver (1995) (see Chiappini et al. 2003b, for details).

We ran two models adopting MM02 nucleosynthetic yields. For the model with $Z=0.004$ only two initial stellar masses ( $3 M_{\odot}$ and $9 M_{\odot}$ ) have been calculated by the authors. The initial conditions are the same as the SR3 and SV3 models, i.e. the same initial gaseous distribution and the same IMF are adopted. The only difference is that the IMF extends up to $60 M_{\odot}$, since MM02, at variance with Woosley \& Weaver (1995), consider the yields coming from stars of initial mass up to $60 M_{\odot}$. Moreover, adopting the MM02 yields, it is not necessary to mix sets of yields coming from different authors, since these authors calculated models in the mass range $[2,60] M_{\odot}$. The results of MM02 have been tested in chemical evolution models (Chiappini et al. 2003b) and are able to reproduce the main feature of the Galaxy in a satisfactory way. We refer as to



Fig. 9. Nitrogen yields in IMS coming from different authors. Squares are the results of RV81; pentagons refer to the VG97 yields. Open triangles show the MM02 models with $Z=10^{-5}$, whereas filled triangles refer to the models with $Z=0.004$.

MM1 for the model with $Z=0.004$ and to MM2 for the one with $Z=10^{-5}$.

The dynamical evolution of models MM1 and MM2 is pretty similar to the one of model SV3: at the end of the simulation the mass content inside the galaxy is $\sim 2 \times 10^{7} M_{\odot}$, thus in agreement with the observations in IZw18. The chemical evolution of these two models is shown in Fig. 10. In spite of the larger production of $\mathrm{N}$ in massive stars, IMS are always required in order to reach the $\mathrm{N}$ values observed in IZw18. It is thus not possible to reproduce the observations assuming that only massive stars contribute to the nitrogen enrichment, as suggested by Izotov \& Thuan (1999; see also next section). The $\mathrm{N}$ produced in model MM1 at the end of the simulation, is remarkably in agreement with the observations in IZw18 and, for a considerable range of time, the model is able to reproduce at the same time the abundances of $\mathrm{C}, \mathrm{N}, \mathrm{O}$, although the O produced by this model lies slightly above the observations. It is however worth reminding that the MM02 yields do not consider the third dredge-up, therefore the final $\mathrm{N}$ abundance should be considered as a lower limit. The MM2 model produces instead much less $\mathrm{N}$ and it cannot reach the observed N/O level. This model can be used to explain the low N/O observed in some Damped Lyman Alpha (DLA) Systems (see Chiappini et al. 2003b).

\subsection{The abundances in the $\mathrm{HI}$ medium}

Two recent papers (Aloisi et al. 2003; Lecavelier des Etangs et al. 2004) tried to determine the composition of the H I gas in IZw18. These two papers agree in pointing out a (slightly) lower metal content in the $\mathrm{H}$ I regions compared to the $\mathrm{H}$ II ones, but the final results of the two papers differ significantly. A direct comparison of these two sets of results is shown in Table 2 .

Aloisi et al. (2003) found an $[\mathrm{O} / \mathrm{Fe}]$ that was low, although with large error-bars $([\mathrm{O} / \mathrm{Fe}]=-0.3 \pm 0.3)$. This very large iron 

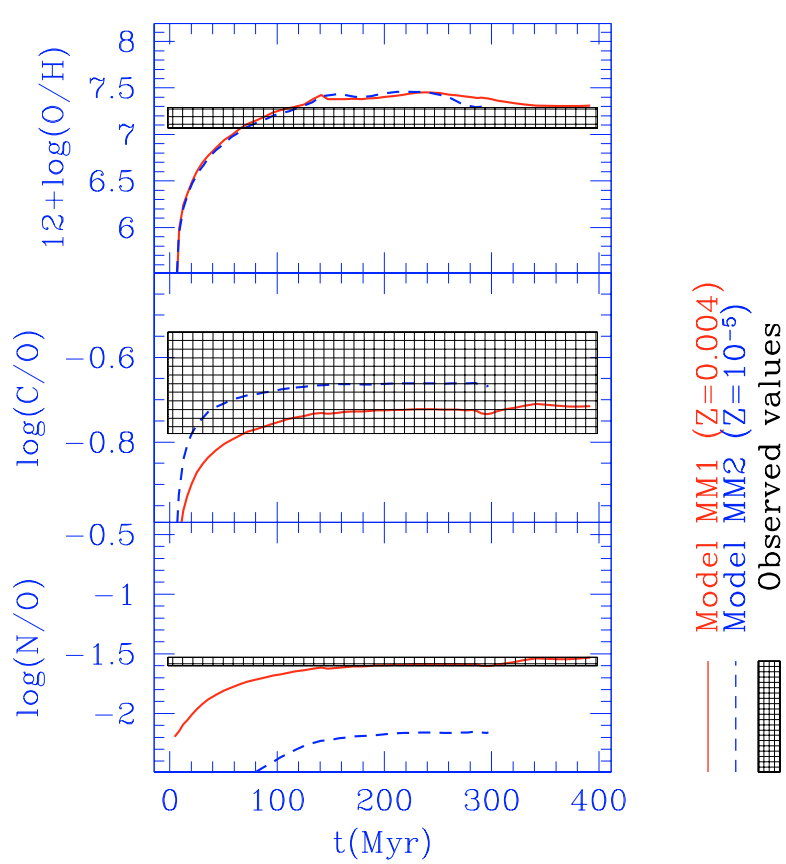

Fig. 10. Evolution of $\mathrm{O}, \mathrm{C}, \mathrm{N}$ for models $\mathrm{MM} 1$ (solid line) and $\mathrm{MM} 2$ (dashed line).

Table 2. Abundances in the H I medium of IZw18.

\begin{tabular}{ccc}
\hline \hline Element ratio & $\mathrm{A03}^{a}$ & $\mathrm{L03}^{b}$ \\
\hline $\log \left(\mathrm{O}_{\mathrm{I}} / \mathrm{H}_{\mathrm{I}}\right)$ & $-5.37 \pm 0.28$ & $-4.7_{-0.6}^{+0.8}$ \\
$\log \left(\mathrm{N}_{\mathrm{I}} / \mathrm{O}_{\mathrm{I}}\right)$ & $-1.54 \pm 0.30$ & $-2.4_{-0.8}^{+0.6}$ \\
$\log \left(\mathrm{Ar}_{\mathrm{I}} / \mathrm{O}_{\mathrm{I}}\right)$ & $-2.38 \pm 0.31$ & $-3.15_{-0.85}^{+0.6}$ \\
\hline
\end{tabular}

${ }^{a}$ Aloisi et al. (2003).

${ }^{b}$ Lecavelier des Etangs et al. (2004).

content in the neutral medium can be explained only by assuming a significant iron production in SNeIa, since it is not possible for SNeII to produce gas with an iron overabundance relative to oxygen. This means that SNeIa already contaminated the surrounding gas at the early phases of the evolution of the galaxy. Aloisi et al. (2003) calculated that $\sim 470$ SNeIa are required in order to justify the iron content of the $\mathrm{H}$ I medium in IZw18. This number is well in agreement with the number of SNeIa exploding in our simulations. Some models of Type Ia SN explosion (Kobayashi et al. 1998; Kobayashi et al. 2000) predict a metallicity threshold for the SNIa occurrence. When the metallicity of the gas $[\mathrm{Fe} / \mathrm{H}]$ is below -1.1 , type Ia SN explosion cannot take place. Actually, the metallicity of IZw18 is well below this threshold, thus, according to this scenario, SNeIa should not be possible.

The comparison between these two sets of results show remarkable differences. In particular, the $\mathrm{O}$ abundance in Lecavelier des Etangs et al. (2004) is consistent with the $\mathrm{O} / \mathrm{H}$ ratio observed in $\mathrm{H}_{\mathrm{II}}$ regions, although the error-bars are huge. Aloisi et al. (2003) show instead an $\mathrm{O}$ abundance a factor of 3-4 lower than in the ionized gas. The difference is due to a different choice of O I lines: Aloisi et al. (2003) measured the



Fig. 11. Evolution of $\mathrm{O}, \mathrm{Fe}, \mathrm{N}$ in the neutral medium for models MM1 (red line) and SR2 (blue line). Vertically dashed area represent the observations (with relative error-bars) performed by Aloisi et al. (2003). Horizontally dashed area refers instead to the observations of Lecavelier des Etangs et al. (2004).

intensity of the line at $\lambda_{0}=1039.23 \AA$. Lecavelier des Etangs et al. (2004), claiming that this line is contaminated by a terrestrial airglow, chose lines at shorter wavelengths $\left(\lambda_{0}=924.95 \AA\right.$, $925.44 \AA, 976.45 \AA$ ). Nitrogen is depleted by a similar factor, thus the N/O ratio is consistent with the observations of the $\mathrm{H}$ II regions. In Lecavelier des Etangs et al. (2004) instead, due to the larger $\mathrm{O}$ content, the $\mathrm{N} / \mathrm{O}$ ratio is much below the observations in the $\mathrm{H}$ II regions.

We are not able to distinguish clearly the $\mathrm{H}_{\mathrm{I}}$ from the $\mathrm{H}_{\text {II }}$ regions in our simulations. Moreover, since the gas in our model is in hydrostatic equilibrium with a potential extending out to $10 \mathrm{kpc}$, the abundance ratios in the neutral medium will depend on what fraction of this gas we decide to consider to calculate the abundance ratios. We decided to select all the gas with a temperature below $7 \times 10^{3} \mathrm{~K}$ in a volume of $\sim 2 \mathrm{kpc} \times 2 \mathrm{kpc}$ in the $R-z$ plane. The resulting evolution of $\mathrm{O}, \mathrm{Fe} / \mathrm{O}$ and $\mathrm{N} / \mathrm{O}$ for model MM1 (solid line) and SR2 (dashed line) is shown in Fig. 11, together with the observations performed by Aloisi et al. (2003, vertically dashed area) and Lecavelier des Etangs et al. (2004, horizontally dashed area). The chemical abundances in the $\mathrm{HI}_{\mathrm{I}}$ and in the $\mathrm{H}_{\mathrm{II}}$ medium as predicted by our models are similar, thus the mixing between these two phases is quite efficient (see also the discussion in $\mathrm{RMD}$ ). The $\mathrm{O}$ predicted by the models seems to be in agreement with the estimates of Lecavelier des Etangs et al. (2004), thus overestimating the oxygen measured by Aloisi et al. (2003). Only Aloisi et al. (2003) measured the iron abundance, which is, as pointed out above, overabundant compared to O. Our models predict an $[\mathrm{O} / \mathrm{Fe}]$ ratio close to solar. Finally, the predictions of model MM1 are in agreement with the 
estimates of the $\mathrm{N} / \mathrm{O}$ ratio in the $\mathrm{H}_{\mathrm{I}}$ regions by Aloisi et al. (2003), whereas the measurements of Lecavelier des Etangs et al. (2004) are well below these predictions.

\section{Discussion}

\subsection{Physical and numerical limitations}

This paper presents the detailed chemical and dynamical evolution of a galaxy ongoing a complex star formation history. It moreover includes a detailed treatment of SNeIa contribution. Few of previous similar studies of star-bursting dwarf galaxies take into consideration these effects. They usually assume a very simple star formation history (in most of the cases an instantaneous burst of star formation) and often neglect the contribution of SNeIa.

Our study is thus a progress in this field, but nevertheless, to properly discuss the results of our simulations, it is important to analyze the limitations of the models. We can distinguish two kinds of limitations: those related to the inaccuracy in reproducing the initial conditions in IZw18 and those related to the code itself, in particular missing physics and numerical resolution problems.

\subsubsection{Initial conditions}

As described in Sect. 3, our model is a 2-D one in which cylindrical coordinates are assumed. The initial gaseous distribution can be seen in RMD, their Fig. 1. This distribution and the way in which the input of energy and metals is considered cannot exactly reproduce the real characteristics of IZw18 for several reasons:

- the distribution of stars in IZw18 is not axially symmetric, as described in Sect. 2. Most of the young stars are concentrated in two star forming regions, whose centers are separated by more than $300 \mathrm{pc}$ (at a distance of 12.6 $\mathrm{Mpc}$, cf. Östlin 2000). Two H II regions are associated to this star forming regions. It is impossible to accurately reproduce this "peanut-shaped" morphology with a 2-D numerical code;

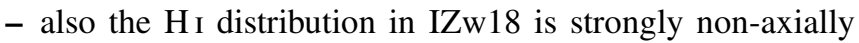
symmetric, with an elongated main component with size $2.2 \times 2.9 \mathrm{kpc}$ and many other patchy structures surrounding this main component (van Zee et al. 1998). Our models cannot reproduce this very complex $\mathrm{H}$ I distribution;

- the energy input rate is very simplified in our model. The energy input rate calculated by the Starburst99 model (Leitherer et al. 1999) shows a much more complex behaviour;

- Wolf-Rayet (WR) stars, although observed in IZw18 (Legrand et al. 1997; de Mello et al. 1998; Brown et al. 2002) are not considered in our simulations. Strong stellar winds coming from these stars may change the energy and metal input rate;

- the energy and metal injection occurs uniformly in a central region. The effect of discrete explosions is neglected (see discussion in RMD) and the possibility of inhomogeneous mixing (Argast et al. 2000) is also excluded. Actually, the metallicity gradient in IZw18 is almost flat in the inner 400-500 pc (Legrand 2000), thus the mixing seems to be rather homogeneous.

\subsubsection{Missing physics}

- Radiative transfer is not included in our code, thus the distinction between the different ISM phases ( $\mathrm{H}_{\mathrm{I}}$ and $\mathrm{H}_{\text {II }}$ regions) is made on the basis of the temperature of the medium.

- A smooth ISM distribution does not represent the correct description of the ISM in galaxies. A significant fraction of gas is in a cloudy phase, which can interact with the diffuse ISM phase through condensation-evaporation processes, changing the thermal balance and possibly also the chemical evolution patterns (Theis et al. 1992; Rieschick \& Hensler 2000). Models including a cloudy phase will be presented in a forthcoming paper (Recchi et al. 2004, in preparation).

- A dusty phase is not included in our code. In spite of the low metallicity content of IZw18, the observed amount of dust is of the order of 2000-5000 $M_{\odot}$ (Cannon et al. 2002), thus a significant fraction of metals is stored in the dusty phase. The metals observed in the $\mathrm{H}_{\mathrm{II}}$ and in the $\mathrm{H}_{\mathrm{I}}$ regions can therefore be only a lower limit of the total amount of metals produced in IZw18.

- Self-gravity is not included in our code. It is assumed that the gravitational potential is dominated by the dark matter halo (fixed in time). Self-gravity may change the behaviour of swept-up shells, due to their large densities. Gravitationally-induced fragmentation may occur, possibly producing star formation in the shell, as suggested by many authors (Larsen et al. 2001; Ehlerová \& Palous 2002).

- Contact discontinuities (the interface between the shocked wind and the shocked ISM) are not properly resolved in any numerical scheme, due to the well-known numerical diffusion problem. In our code the contacts are spread over 4-8 zones. Inaccuracy in the treatment of contacts and poorness in the spatial resolution (in our code the central resolution is $5 \mathrm{pc}$ and the size ratio between adjacent zones is 1.03) may lead to wrong determinations of the cooling time-scale of metals. Actually, recent hydrodynamical simulations (de Avillez \& MacLow 2002; Marcolini et al. 2004) show that the amount of cooled ejecta is only poorly sensitive to the numerical resolution of the simulation. A discussion of this point is also addressed in Sect. 3.2 of RMD.

Some of the limitations will be resolved in forthcoming papers, some others are harder to treat. However, it is worth noting that IZw18 shows almost uniform properties (i.e. no significant metallicity gradients, marginal uniformity in the metallicities of the NW and of the SE region; see e.g. Vílchez \& Iglesias-Páramo 1998; Legrand 2000), thus the comparison of the results of our models with the global properties of the main body of IZw18 is meaningful. 


\subsection{The evolutionary history of $I Z w 18$}

What can we infer about the evolutionary history of IZw18 from these chemical and dynamical simulations? First of all in our models we do not need stars older than $300 \mathrm{Myr}$ to justify the main chemical and dynamical properties of IZw 18 . Observations of the stellar populations in nearby Local Group galaxies show the presence of old stars (i.e. with an age of several Gyr) in all the dwarf galaxies, of both late and early type (see e.g. Grebel 1997). In the galaxies in which the spectroscopy and photometry is deep enough, the presence of old stars is thus ubiquitous. If we go further, outside the Local Group, we start looking at objects in which the presence of older stars is more questionable. One of the best examples of young galaxies is SBS 0335-052, a nearby, very metal-poor BCD galaxy. Vanzi et al. (2000) showed that the NIR emission is dominated by very young $(t \lesssim 5 \mathrm{Myr})$ stars and no need for older stars has been invoked. NGC 1569, another wellknown nearby dwarf galaxy, is a post-starburst galaxy dominated by a recently formed, perhaps still forming, star cluster system (Anders et al. 2004), although evidence for the presence of older stars has been observed (Vallenari \& Bomans 1996; Greggio et al. 1998; Aloisi et al. 2001).

As described in Sect. 2, the age of IZw18 has been questioned for many years. It is nowadays accepted that IZw18 is not a galaxy experiencing star formation for the first time. An intermediate-age population of stars is present. Optical spectroscopy and photometry is not deep enough to resolve stars on the main sequence, allowing us only to go down to a look-back time of the order of 1 Gyr (ATG; Östlin 2000), so stars older than $1 \mathrm{Gyr}$, if any, would be undetected. As described in Sect. 2, the method of color fitting developed by Hunt et al. (2003) allows the authors to put constraints on the mass of stars older than $\sim 500$ Myr. These stars do not contribute more than $22 \%$ to the total mass in stars of IZw18.

From a chemo-dynamical point of view we note that an episode of star formation lasting for $\sim 300 \mathrm{Myr}$ produces a very large amount of $\mathrm{N}$, synthesized in a primary way in intermediate-mass stars. As can be seen in Fig. 7, the N/O ratio in a continuous burst model is almost stable after $\sim 100 \mathrm{Myr}$. This is due to the fact that the nitrogen continuously produced by the model is ejected outside the galaxy more easily than the oxygen, therefore it does not accumulate inside the galaxy. Only a very long quiescent period can completely quench the $\mathrm{O}$ production, resulting in an increase of the $\mathrm{N} / \mathrm{O}$ ratio, up to the moment in which a second burst of star formation releases a large amount of $\mathrm{O}$ into the ISM, thus drastically reducing the N/O (see Fig. 3, dashed line). An episode of star formation lasting more than $300 \mathrm{Myr}$ is therefore acceptable from the chemical point of view.

From a dynamical point of view, in spite of the low level of star formation, the energy produced and restored into the ISM by $\mathrm{SN}$ explosions and stellar winds is very large and able to unbind a significant fraction of the ISM. As described in Sect. 3, the energy feedback depends on some poorly constrained parameters, in particular on the thermalization efficiency. Our values of the thermalization efficiencies are calculated in a self-consistent way starting from the average values of temperature and density in the region in which the feedback occurs. Nevertheless, the physics of the interaction of the Supernovae with the surrounding ISM is poorly known and the thermalization efficiency should be considered as a free parameter. The adopted thermalization efficiency for SNeIa is large and in the "real world" this value can be also significantly lower (although the average explosion energy of SNeIa should be a little larger than the adopted value of $10^{51} \mathrm{erg}$ ). As seen in Sect. 4.3 , a very low SNeIa thermalization efficiency helps in keeping a larger fraction of ISM bound in the galactic potential well. The adopted thermalization efficiency for SNeII is instead very low. Strickland \& Stevens (2000) for example adopted a thermalization efficiency equal to unity for SNeII. Thus in a galaxy like IZw18, a constant star formation rate, even at a low level, cannot be sustained for more than a few hundred Myr, unless a very low thermalization efficiency for both SNeII and SNeIa is adopted. A flatter IMF, as suggested by ATG, would result in a much higher energy production rate, so it would easily unbind the gas in the galaxy. A possible alternative solution is an upper cut-off in the IMF mass range well below $40 M_{\odot}$ (Kroupa \& Weidner 2003; Tolstoy \& Venn 2003). A paper based on a set of models of this kind is in preparation.

\section{Conclusions}

In this paper, we have computed the chemical and dynamical evolution of a galaxy similar to IZw18 under the assumption of a continuous, weak burst of star formation lasting for $270 \mathrm{Myr}$ and a 5 times stronger burst occurring more recently and lasting for $5 \mathrm{Myr}$. Such a star formation history has been suggested by Aloisi et al. (1999) in order to reproduce the observed CMD of IZw18. We have tested different IMF slopes and different sets of yields for intermediate-mass stars and massive ones. Different initial gaseous distributions are also tested. In most of the explored cases, a galactic wind develops, as a consequence of the energy produced by SNeII, SNeIa and winds from intermediate-mass stars. The time-scale for the development of the galactic wind depends mostly on the IMF slope which can change the energy input rate by an order of magnitude. The mass of gas initially present inside the galaxy influences also the development of a galactic wind, but not significantly.

Our main conclusions can be summarized as follows:

- At variance with models with instantaneous bursts of star formation, in which the variations in the abundance ratios occurred on very short time-scales, thus matching the observed values of IZw18 only for small intervals of time (see dotted and dashed lines in Fig. 3), in these continuous star formation burst models, the chemical composition of the gas is stable for longer time-scales. However, models with a Salpeter (1955) IMF, when the IMS yields of van den Hoek \& Groenewegen (1997) or Renzini \& Voli (1981) are implemented, overestimate the $\mathrm{N}$ composition of the ISM by a factor of 2-3. It is therefore possible to match the observations of the N/O ratio in $\mathrm{IZw} 18$ by reducing the yields from the above authors by the same factor (see long-dashed line in Fig. 3). Due to the uncertainties in the computation of the 
$\mathrm{N}$ yield, this reduction cannot be ruled out. Models with a steep ( $x=1.7)$ IMF largely overestimate $\mathrm{N}$ and underestimate O. Finally, models with a flat $(x=0.5)$ IMF slightly overestimate $\mathrm{O}$ and underestimate $\mathrm{N}$. These flat IMF models, although hardly acceptable from a dynamical point of view (they inject too much energy into the gas), can be fine tuned and can be the most promising models, if Renzini \& Voli (1981) or van den Hoek \& Groenewegen (1997) yields are implemented, in order to reproduce the chemical properties of IZw18.

- New models of IMS and massive stars evolution with rotation (Meynet \& Maeder 2002) predict a larger N primary production in massive stars. Since they do not reach the hot-bottom burning phase, they also have a significantly reduced production of nitrogen in IMS. By means of these yields, it is possible to reproduce the abundances of the ISM in IZw18 in a satisfactory way.

- Our present models confirm the existence of the so-called differential winds, namely the freshly produced metals are ejected more easily than the pristine gas. In agreement with the results of Recchi et al. (2001), the metals with the largest ejection efficiencies are the ones produced on longer time-scales (Fe and $\mathrm{N}$ in particular), since, when the bulk of these metals is produced, a galactic tunnel has been already carved and it is easy for the galactic wind to carry them outside the galaxy. From a chemical point of view, the consequence of this selective wind is an $[\alpha / \mathrm{Fe}]$ ratio in the gas outside the galaxy lower than in the gas inside. This difference is evident but much smaller than the one produced in models with a single, instantaneous burst of star formation (Recchi et al. 2001). Nevertheless, also in this case SNeIa, often ignored in previous similar works, play a decisive role in the evolution of BCD galaxies. This selective wind can also explain the large amount of $\mathrm{Fe}$ and the very low $[\alpha / \mathrm{Fe}]$ ratio observed in the Intracluster Medium, if the same mechanism acts in elliptical galaxies which are the main contributors to metals in the ICM (see Pipino et al. 2002).

- The chemical composition of the H I gas can also be inferred from our models. The resulting abundances and abundance ratios are fairly well in agreement with the results obtained in the HiI regions, showing that the mixing between these two phases is efficient and fast. In fact, mostly due to the low thermalization efficiency of SNeII, the cooling time-scale of freshly produced metals is short (of the order of a few tens of Myr). The "instantaneous mixing" assumption, widely adopted in chemical evolution models, is therefore acceptable. The results of the chemical evolution in the $\mathrm{H}_{\mathrm{I}}$ medium can be compared with recent observations of the $\mathrm{H}_{\mathrm{I}}$ regions of IZw18 performed with FUSE (Aloisi et al. 2003; Lecavelier des Etangs et al. 2004). This comparison does not allow us to impose robust constraints, since the results of these two groups are remarkably different.

Acknowledgements. We thank the anonymous referee for many comments and suggestions which improved the paper. We are grateful to Cristina Chiappini for useful comments and for having provided us interpolations to the yields of Meynet \& Maeder (2002) and Gerhard Hensler for stimulating discussions. S.R. acknowledges generous financial support from the Alexander von Humboldt Foundation and Deutsche Forschungsgemeinschaft (DFG) under grant HE 1487/28-1. F.M., S.R and A.D'E. acknowledge financial support from the INAF (Italian National Institute for Astrophysics) contract PRIN2002 by the title "Blue Compact Galaxies: primordial helium and chemical evolution".

\section{References}

Aloisi, A., Clampin, M., Diolaiti, E., et al. 2001, AJ, 121, 1425

Aloisi, A., Savaglio, S., Heckman, T. M., et al. 2003, ApJ, 595,760

Aloisi, A., Tosi, M., \& Greggio, L. 1999, AJ, 118, 302 (ATG)

Anders, P., de Grijs, R., Fritze-v. Alvensleben, U., \& Bissantz, N. 2004, MNRAS, 347, 17

Anders, E., \& Grevesse, N. 1989, Geochim. Cosmochim. Acta, 53, 197

Argast, D., Samland, M., Gerhard, O. E., \& Thielemann, F.-K. 2000, A\&, 356, 873

Brown, T. M., Heap, S. R., Hubeny, I., Lank, T., \& Linder, D. 2002 , ApJ, 579, L75

Cannon, J. M., Skillman, E. D., Garnett, D. R., \& Dufour, R. J. 2002, ApJ, 565, 931

Carigi, L. 2000, Rev. Mex. Astron. Astrofis., 36, 171

Chiappini, C., Romano, D., \& Matteucci, F. 2003a, MNRAS, 339, 63

Chiappini, C., Matteucci, F., \& Meynet, G. 2003b, A\&A, 410, 257

Davidson, K., \& Kinman, T. D. 1985, ApJS, 58, 321

Davidson, K., Kinman, T. D., \& Friedman, S. D. 1989, AJ, 97, 1591

de Avillez, M., \& MacLow, M.-M. 2002, ApJ, 581, 1047

de Mello, D. F., Schaerer, D., Heldmann, J., \& Leitherer, C. 1998, ApJ, 507, 199

D'Ercole, A., \& Brighenti, F. 1999, MNRAS, 309, 941

De Young, D. S., \& Heckman, T. M. 1994, ApJ, 431, 598

Dufour, R. J., Garnett, D. R., \& Shields, G. A. 1988, ApJ, 332, 752

Dufour, R. J., \& Hester, J. J. 1990, ApJ, 350, 149

Dufour, R. J., Esteban, C., \& Castañeda, H. O. 1996, ApJ, 471, L87

Ehlerová, S., \& Palous, J. 2002, MNRAS, 330, 1022

Fall, S. M. 2004, in Formation and Evolution of Massive Young Star Clusters, ed. H. J. G. L. M. Lamers, A. Nota, \& L.J. Smith (San Francisco: ASP), 2004, in press

French, H. B. 1980, ApJ, 240, 41

Fryer, C. L. 1999, ApJ, 522, 413

Fryer, C. L., \& Kalogera, V. 2001, ApJ, 554, 548

Garnett, D. R., Dufour, R. J., Peimbert, M., et al. 1995, ApJ, 471, L87

Garnett, D. R., Skillman, E. D., Dufour, R. J., \& Shields, G. A. 1997, ApJ, 481, 174

Grebel, E. K. 1997, Rev. Mod. Astron. 10, 29

Greggio, L., \& Renzini, A. 1983, A\&A, 118, 217

Greggio, L., Tosi, M., Clampin, M., et al. 1998, ApJ, 504, 725

Henry, R. B. C., Edmunds, M. G., \& Köppen, J. 2000, ApJ, 541, 660

Hirashita, H., Hunt, L. K., \& Ferrara, A. 2002, MNRAS, 330, L19

Holweger, H. 2001, in Joint SOHO/ACE Workshop, Solar and Galactic Composition, ed. R.F. Wimmer-Schweingruber (New York: Am. Inst. Phys.), Am. Inst. Phys. Conf. Proc., 598, 23

Hunt, L. K., Thuan, T. X., \& Izotov, Y. I. 2003, ApJ, 588, 281

Hunter, D. A., \& Thronson, H. A. 1995, ApJ, 452, 238 
Izotov, Y. I., Foltz, C. B., Green, R. F., Guseva, N. G., \& Thuan, T. X. 1997a, ApJ, 487, L37

Izotov, Y. I., \& Thuan, T. X. 1999, ApJ, 511, 639

Izotov, Y. I., Thuan, T. X., \& Lipovetsky, V. A. 1997b, ApJS, 108, 1

Kinman, T. D., \& Davidson, K. 1981, ApJ, 243, 127

Kobayashi, C., Tsujimoto, T., Nomoto, K., Hachisu, I., \& Kato, M. 1998, ApJ, 503, L155

Kobayashi, C., Tsujimoto, T., \& Nomoto, K. 2000, ApJ, 539, 26

Koo, B. C., \& McKee, C. F. 1992, ApJ, 388, 9

Kroupa, P. 2002, Science, 295, 82

Kroupa, P., \& Weidner, C. 2003, ApJ, 598, 1076

Kroupa, P., Tout, C. A., \& Gilmore, G. 1990, MNRAS, 244, 76

Kunth, D., Lequeux, J., Sargent, W. L. W., \& Viallefond, F. 1994, A\&A, 282, 709

Kunth, D., Matteucci, F., \& Marconi, G. 1995, A\&A, 297, 634

Kunth, D., \& Östlin, G. 2000, A\&AR, 10, 1

Kunth, D., \& Sargent, W. L. W. 1983, ApJ, 273, 81

Larsen, S. 2002, AJ, 124, 1393

Larsen, T. I., Sommer-Larsen, J., \& Pagel, B. E. J. 2001, MNRAS, 323,555

Lecavelier des Etangs, A., Désert, J.-M., Kunth, D., et al. 2004, A\&A, 413,131

Legrand, F. 2000, A\&A, 354, 504

Legrand, F., Kunth, D., Roy, J.-R., Mas-Hesse, J. M., \& Walsh, J. R. 1997, A\&A, 326, L17

Legrand, F., Kunth, D., Roy, J.-R., Mas-Hesse, J. M., \& Walsh, J. R. 2000, A\&A, 355, 891

Legrand, F., Tenorio-Tagle, G., Silich, S., Kunth, D., \& Cerviño, M. 2001, ApJ, 560, 630

Leitherer, C., Schaerer, D., Goldader, J. D., et al. 1999, ApJS, 123, 3

Lequeux, J., Peimbert, M., Rayo, J. F., Serrano, A., \& Torres-Peimbert, S. 1979, A\&A, 80, 155

Lequeux, J., \& Viallefond, F. 1980, A\&A, 91, 269

Levshakov, S. A., Kegel, W. H., \& Agafanova, I. I. 2001, A\&A, 373, 836

MacLow, M.-M., \& Ferrara, A. 1999, ApJ, 513, 142

Marcolini, A., Brighenti, F., \& D'Ercole, A. 2004, MNRAS, 352, 363

Martin, C. L. 1996, ApJ, 465, 680

Martin, C. L., Kobulnicky, H. A., \& Heckman, T. M. 2002, ApJ, 574, 663

Mas-Hesse, J. M., \& Kunth, D. 1999, A\&A, 349, 765

Matsumoto, T., \& Hanawa, T. 2003, ApJ, 595, 913

Matteucci, F. 1986, MNRAS, 221, 911

Matteucci, F. 1996, Fund. Cosm. Phys., 17, 283

Matteucci, F., \& Greggio, L. 1986, A\&A, 154, 279

Matteucci, F., \& Recchi, S. 2001, ApJ, 558, 351

Melioli, C., \& de Gouveia Dal Pino, E. M. 2004, A\&A, 424, 817

Meurer, G. R., Heckman, T. M., Leitherer, C., et al. 1995, AJ, 110, 2665

Meynet, G., \& Maeder, A. 2002, A\&A, 390, 561 (MM02)
Nomoto, K., Maeda, K., Umeda, H., et al. 2003, in A Massive Star Odissey, from Main Sequence to Supernova, ed. K. A. van der Hucht, A. Herrero \& C. Esteban (ASP), Proc. IAU Symp., 212,395

Östlin, G. 2000, ApJ, 535, L99

Pagel, B. E. J., Simonson, E. A., Terlevich, R. J., \& Edmunds, M. G. 1992, MNRAS, 255, 325

Papaderos, P., Izotov, Y. I., Thuan, T. X., et al. 2002, A\&A, 393, 461

Pettini, M., \& Lipman, K. 1995, A\&A, 297, L63

Pipino, A., Matteucci, F., Borgani, S., \& Biviano, A. 2002, NewA., 7, 227

Recchi, S., Matteucci, F., \& D'Ercole, A. 2001, MNRAS, 322, 800 (RMD)

Recchi, S., Matteucci, F., D’Ercole, A., \& Tosi, M. 2002, A\&A, 384, 799 (R02)

Renzini, A., \& Voli, M. 1981, A\&A, 94, 175 (RV81)

Rieschick, A., \& Hensler, G. 2000, in ASP Conf. Ser. Cosmic Evolution and Galaxy Formation: Structure, Interactions and Feedback, ed. J. Franco, E. Terlevich, O. Lòpez-Cruz, \& I. Arextaga (San Francisco: ASP), 130

Salpeter, E. E. 1955, ApJ, 121, 161

Sargent, W. L. W., \& Searle, L. 1970, ApJ, 162, L155

Scalo, J. M. 1986, Fund. Cosm. Phys., 11, 1

Searle, L., \& Sargent, W. L. W. 1972, ApJ, 173, 25

Silich, S., \& Tenorio-Tagle, G. 1998, MNRAS, 299, 249

Skillman, E. D., \& Kennicutt, R. C. 1993, ApJ, 411, 655

Strickland, D. K., \& Stevens, L. R. 1999, MNRAS, 306, 43

Strickland, D. K., \& Stevens, L. R. 2000, MNRAS, 314, 511

Summers, L. K., Stevens, I. R., Strickland, D. K., \& Heckman, T. M. 2003, MNRAS, 342, 690

Takeuchi, T. T., Hirashita, H., Ishii, T. T., Hunt, L. K., \& Ferrara, A. 2003, MNRAS, 343, 839

Theis, C., Burkert, A., \& Hensler, G. 1992, A\&A, 265, 465

Thuan, T. X., Izotov, Y. I., \& Lipovetsky, V. A. 1995, ApJ, 445, 108

Timmes, F. X., Woosley, S. E., \& Weaver, T. A. 1995, ApJS, 98, 617

Tolstoy, E., \& Venn, K. 2003, in Highlights of Astronomy, 13, ed. O. Engvold (ASP), Proc. IAU Symp., 217, to be published

Vallenari, A., \& Bomans, D. J. 1996, A\&A, 313, 713

van den Hoek, L. B., \& Groenewegen, M. A. T. 1997, A\&AS, 123, 305 (VG97)

van Zee, L., Westpfahl, D., Haynes, M., \& Salzer, J. J. 1998, AJ, 115, 1000

Vanzi, L., Hunt, L. K., Thuan, T. X., \& Izotov, Y. I. 2000, A\&A, 363, 493

Vílchez, J. M., \& Iglesias-Páramo, J. 1998, ApJ, 508, 248

Vorobyov, E. I., Klein, U., Shchekinov, Yu. A., \& Ott, J. 2004, A\&A, 413, 939

Weaver, R., McCray, R., Castor, J., Shapiro, P., \& Moore, R. 1977, ApJ, 218, 377

Woosley, S. E., \& Weaver, T. A. 1995, ApJS, 101, 181

Zwicky, F. 1966, ApJ, 143, 192 